\title{
PERIODICALLY REGULAR CHORDAL RINGS ARE PREFERABLE TO DOUBLE-RING NETWORKS
}

\author{
BEHROOZ PARHAMI \\ Dept. of Electrical \& Computer Engineering \\ University of California, Santa Barbara, CA 93106-9560, USA \\ Phone: +18058933211 \\ Fax: +18058933262 \\ phomiogcencshed
}

Received 15 September 2007

Accepted 20 June 2008

\begin{abstract}
The susceptibility of ring networks to disconnection as a result of one or two node/link failures has led to a number of proposals to increase the robustness of such networks. One class of proposals, discussed primarily in the networking and communication communities, but also advocated for use in parallel and distributed systems, involves the provision of a second ring to improve system throughput during nomal operation and to make alternate paths available in the event of node or link failures. With regard to the advantages just listed, chordal rings are quite similar to double-ring networks, and periodically regular chordal (PRC) rings offer the added benefit of smaller node degree compared with node-symmetric chordal rings of comparable diameters. In this paper, we note that certain double-ring networks are isomorphic to suitably constructed PRC rings, while other varieties correspond to PRC rings that closely approximate their static and dynamic attributes. These results, combined with greater flexibility and other advantages for the PRC-ring family of networks, demonstrate that PRC rings are preferable to double-ring networks in virtually all application contexts. A byproduct of our observations on the relationships among double-ring networks, generalized Petersen graphs, and PRC rings is that by amalgamating known results for these network classes, many more tools and techniques become applicable to the analysis and synthesis of robust ring networks for parallel and distributed computing. As examples of new results that can be developed with this viewpoint, we present near-optimal and fault-tolerant routing algorithms for our PRC ring networks.

Keywords:: Bisection width, Chordal ring, Connectivity, Diameter, Embedding, Fault diameter, Fault tolerance, Hierarchical network, Loop, Optimal routing, Ring, Symmetric network.
\end{abstract}




\section{Introduction}

The popularity of the ring topology (Fig. 1a) for many communications and parallel processing applications stems from its structural simplicity and very efficient routing protocols. These properties contribute to low implementation cost and high transmission throughput, with high latency being a potential drawback. Even though a single node or link failure will not disconnect the nodes of an $n$-node ring $R(n)$, it has an effect on the routing strategy and tends to further increase the transmission latency. More importantly, two failures (node/node, link/link, or node/link) will almost certainly isolate some parts of the network. Additionally, if the ring's channels (metal wires or optical links) are laid within one linear trench, a single cable-cut accident, which is known to be a primary source of failures in communication networks [16], can have the same disruptive effect as two node/link failures.

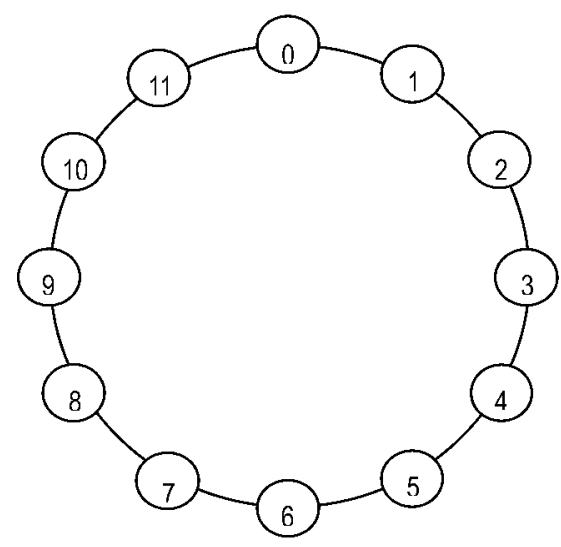

(a) Simple ring, $R(12)$

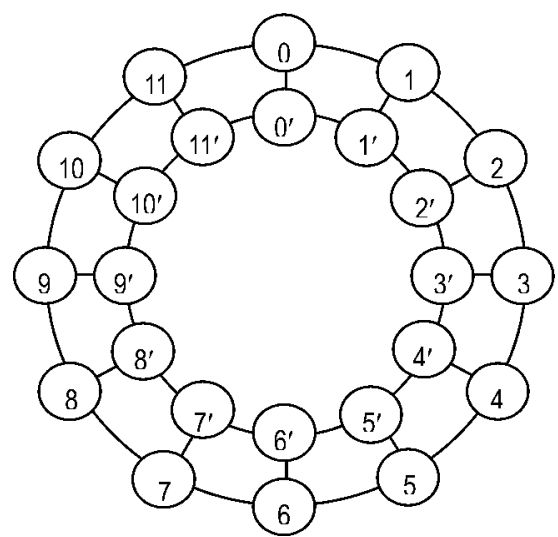

(c) Double ring, $D R(12 ; 1)$

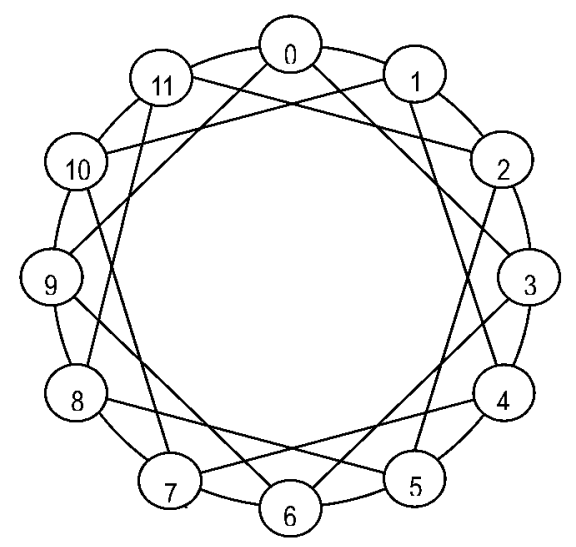

(b) Chordal ring, $C R(12 ; 3)$

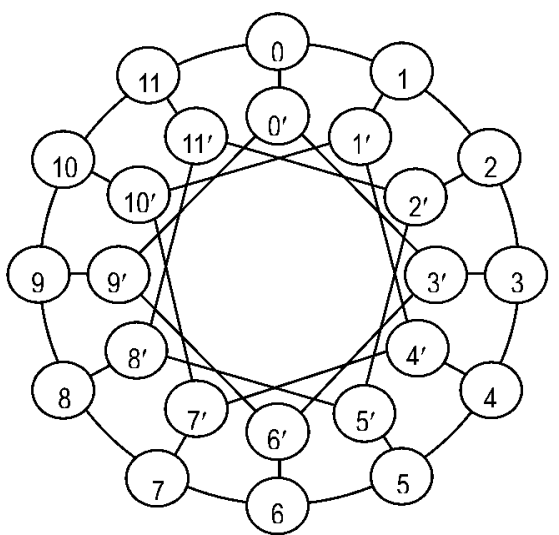

(d) Generalized Petersen graph, $G P(12 ; 3)$

Fig. 1. Examples of ring, chordal ring, double ring, and generalized Petersen graph. 
Table I. List of Notation and Abbreviations.

\begin{tabular}{ll}
\hline \pm 1 & Forward and backward ring links \\
$\pm s$ & Forward and backward skip links \\
$C R\left(p ; s_{1}, s_{2}, \ldots, s_{k}\right)$ & Chordal ring with $p$ nodes and $k$ skip distances, as listed \\
$D$ & Network diameter \\
$D_{\mathrm{R}}$ & Diameter according to the routing algorithm $\mathrm{R}$ \\
$D R(n ; s)$ & Double-ring network with $2 n$ nodes and skip distance $s$ \\
$g$ & Group size in a PRC ring \\
$G P(n ; s)$ & General Petersen graph with $2 n$ nodes and skip distance $s$ \\
$N 2 R(n ; s)$ & Another name for $D R(n ; s)$ \\
$p$ & Number of nodes or processors \\
$P$ & Petersen graph; see Fig. 2 \\
PRC ring & Periodically regular chordal ring \\
$P R C_{3}(p ; s)$ & Degree-3 chordal ring with $p$ nodes and skip distance $s$ \\
$R(q)$ & Ring network with $q$ nodes \\
$s, s_{i}$ & Skip distance(s)
\end{tabular}

One solution that has been proposed for increasing the robustness of ring networks entails the addition of skip links or chords into a $p$-node chordal ring structure $C R\left(p ; s_{1}, s_{2}, \ldots, s_{k}\right)$, where the skip distances or chord lengths $s_{i}$ imply that each node $j$ is connected to nodes $j \pm s_{i}$, besides the ring links that connect it to $j \pm 1$ (Fig. 1b). Another proposed solution is the introduction of a secondary ring to form a double-ring network (Fig. 1c). Combining the two ideas of secondary ring and chordal connection leads to the generalized Petersen graph (Fig. 1d) in which the inner ring has skip links instead of ordinary ring connections. A special case of $G P(n ; s)$, the generalized Petersen graph with $p=2 n$ nodes and skip distance $s$, is the generalized double-loop network which restricts $n$ and $s$ to be relatively prime. The double-ring network $D R(n ; s)$, exemplified by Fig. 1c, improves the robustness attributes of the network but has a small impact on performance, whereas the variations in Figs. $1 \mathrm{~b}$ and $1 \mathrm{~d}$ can potentially have significant effects on the network diameter, average internode distance, and bisection width, and thus on the communication performance.

We label the nodes of a $q$-node ring from $v_{0}$ to $v_{q-1}$, or simply 0 to $q-1$, and say that node $j$ is linked to nodes $j-1$ and $j+1$. Throughout our discussions, $p$ denotes the total number of nodes, so that various architectures can be easily compared against each other. For a generalized Petersen graph or generalized double-ring network, with $p=2 n$ nodes, the outer nodes $u_{0}$ to $u_{n-1}$ and inner nodes $v_{0}$ to $v_{n-1}$ are connected by the following edges for $0 \leq j \leq n-1$ : 
Outer edges $\left(u_{j}, u_{j+1}\right)$

Inner edges $\left(v_{j}, v_{j+s}\right)$

Spoke edges $\left(u_{j}, v_{j}\right)$

In this paper, all node-index expressions within a ring are taken to be modular, with the node having the largest index being adjacent to node 0 of the same ring. Thus, for example, in the case of the outer edges defined above, if $u_{j}=u_{n-1}$, then $u_{j+1}$ is taken to be $u_{0}$. Table I contains a list of key notation and abbreviations used throughout the paper.

\section{Related Work}

A number of variations on the simple directed or undirected ring networks have been studied in the literature on parallel and distributed computing or deployed in actual systems. The most obvious of these, the provision of a secondary ring in a double-ring structure (Fig. 1c), yields greater robustness and some improvement in performance with minimal increase in node cost (one extra link per node). To avoid any confusion owing to loose or inconsistent terminology in the literature, we note that "double-ring" or "double-loop" network has been used to describe a variety of other ring-based systems, including simple bidirectional rings (their clockwise and counterclockwise links can be viewed as forming separate rings) and a generalized form of the above [35] in which forward links are as in an ordinary ring and backward links lead to nodes that are further away ("forward-loop backward-hop networks").

As rings that are augmented to overcome the main drawback of wide diameter and long average internode distance, chordal rings have been studied extensively for use as communication and parallel processing networks [2], [8], [19], [20], [30]. Applications of chordal rings to parallel systems started very early in the history of parallel processing and have continued to date [18], [38], although in some cases the interconnection structures include subtle variations and carry different names, thus making it difficult to identify the underlying chordal ring networks. The bulk of studies of chordal rings in relation to interconnection networks deal with networks of small, fixed node degrees; most commonly, 3-6 for undirected (4 being most heavily studied [5], [9], [11]), and 2-3 for directed networks [34], [35]. The rich mathematical properties of chordal rings has also attracted numerous theoretical studies, some without explicit or immediate applications. For example, chordal rings figure prominently in many attempts to generate graphs having small diameter or average distance, maximal connectivity, and other graph-theoretic properties [6], [7], [10], [14], [26], quests that present many hard (in the sense of complexity theory) and open problems.

Some of the advantages of chordal rings persist when special pruning schemes are applied to convert them from completely regular to periodically regular, with a small, 
fixed node degree [28]. This serves as a mechanism for generating fixed-degree networks with desirable properties mirroring those of more densely connected chordal rings. On the negative side, determination of diameter and other topological parameters of chordal rings can be quite difficult [12], [39], [40]. Even for restricted classes of chordal rings such as the ones with a single skip type (degree 4), determination of topological properties is nontrivial in general and the problems have not yet been completely solved [13].

In parallel with the studies by computer scientists and engineers on variants of double-ring networks, mathematicians have considered similar structures from a graph theoretic point of view. The starting point for these studies is the Petersen graph, depicted in Fig. 2, which is the largest graph of degree 3 and diameter 2, or alternatively, the lowest-diameter network that can be built out of 10 degree- 3 nodes. The Petersen graph is ubiquitous in graph theory, given that it frequently shows up as exception or counterexample to many otherwise valid results [17]. The observation that the Petersen graph $P$ of Fig. 2a consists of an outer ring, an inner ring, and spokes connecting the two, has led to formulating the generalization $G P(n ; s)$, defined in Section 1 and exemplified by Fig. 1d.

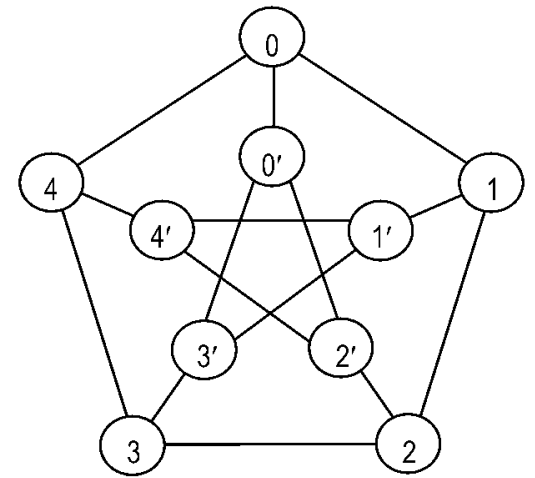

(a) The Petersen graph $P \equiv G P(5 ; 2)$

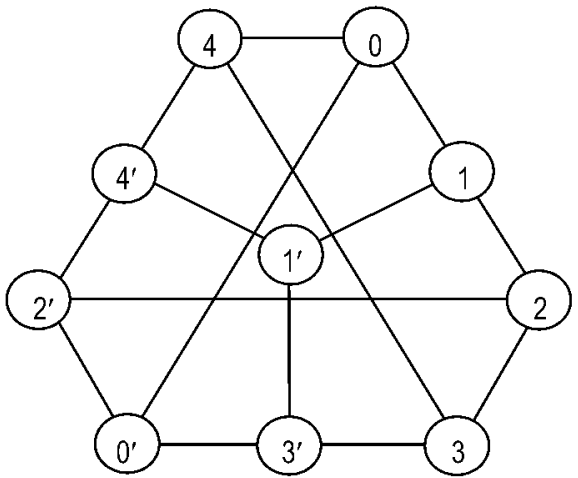

(b) Another drawing of $P$

Fig. 2. The ubiquitous Petersen graph $P$.

Among known results [1] for the generalized Petersen graph $\operatorname{GP}(n ; s)$ is the fact that it is almost always Hamiltonian (i.e., contains or embeds a $2 n$-node cycle), the only exceptions occurring for $G P(n ; 2)$ when $n=5 \bmod 6$ (see Theorem 13.1 on p. 316 of [17]). The non-Hamiltonicity of $P$, for example, can be readily seen from its representation in Fig. 2b, by visualizing options for a Hamiltonian path as it enters and leaves the center node $1^{\prime}$. Another useful known mathematical property is that for $n$ odd, 
$G P(n ; s)$ is isomorphic to $G P(n ;\lceil n / 2\rceil+1-s)$, so that one need only consider $G P(n ; s)$ for $s \leq\lceil n / 4\rceil$ when $n$ is odd. Besides the Petersen graph $P \equiv G P(5 ; 2)$, other noteworthy special cases of $G P(n ; s)$ include the cubical graph $G P(4 ; 1)$, the Möbius-Kantor graph $G P(8 ; 3)$, the Desargues graph $G P(10 ; 3)$, the symmetric cubic graph $F_{24} \equiv G P(12 ; 5)$, and the prism graph $G P(n ; 1)$.

The double-ring network of Fig. 1c, also called the prism graph as noted above, was first proposed to improve the robustness of a simple ring, which is particularly vulnerable to cable-cut accidents when deployed in underground trenches. Pedersen and coworkers [21], [31], [32] studied generalized double-ring networks (N2R networks, in their terminology) in comparison with simple double-ring networks and degree-3 chordal rings, taking topological parameters, such as diameter and average internode distance, as well as reliability into consideration. Their results, which are experimental and obtained by way of extensive computer simulation, do not represent any advance in our theoretical understanding generalized Petersen graphs. However, they do point to potential advantages of $\operatorname{DR}(n ; s)$ and $G P(n ; s)$ in comparison with other networks of similar cost and complexity.

\section{Periodic Regularity}

In the rest of this paper, we aim to reveal some properties of double-ring networks. The key observation pertains to isomorphism relationships between $G P(n ; s)$ and periodically regular chordal rings, or PRC rings for short [28]. Thus, we include in this section a capsule review of the latter. Discovery of isomorphisms between interconnection networks has played a key role throughout the history of parallel processing. Many classes of networks, proposed independently, were subsequently discovered to be isomorphic or homomorphic. The most well-known examples are equivalence and containment results among butterfly, shuffle-exchange, and cube-connected cycles networks. Far from making the independent derivations of these networks worthless, such results actually helped create alternate views that facilitated understanding for structural studies and algorithm development.

Consider a $p$-node ring. It can be converted to a chordal ring by adding $g$ skip links (chords) of lengths $s_{1}<s_{2}<\cdots<s_{g}$ (with $1=s_{0}<s_{1}$ ) to each node, so that any node $j$ is connected to the nodes $j \pm s_{i}$, for $0 \leq i \leq g$. The result is a $p$-node network of node degree $2 g+2$ and with $p(g+1)$ links. Now, in order to reduce the node degree of such a network, while maintaining some of its desirable attributes such as small diameter and average internode distance, we may pursue the following strategy. Let $g$ divide $p$. Rather than provide all $g$ different skip links for each node, we divide the nodes into $g$ classes 
according to the residue of their indices modulo $g$. We assign a single skip link type to each class of nodes. As shown in Fig. 3, node $j$ may be provided with two skip links to nodes $j \pm s_{g-(j \bmod g)}$. The node degree is thus reduced from $2 g+2$ to 4 and the number of links from $p(g+1)$ to $2 p$. Both the diameter and average internode distance increase by no more than $g$ as a result of this strategy, which constitutes a form of pruning. Given that $g$ is relatively small in practice, the loss of performance due to the increased routing distances is negligible (aggregate network bandwidth is a different story, of course). Certain conditions on the skip distances are required if the resulting network is to be regular with regard to node degree. However, here we are not interested in PRC rings in their full generality, so there is no need to discuss such conditions.

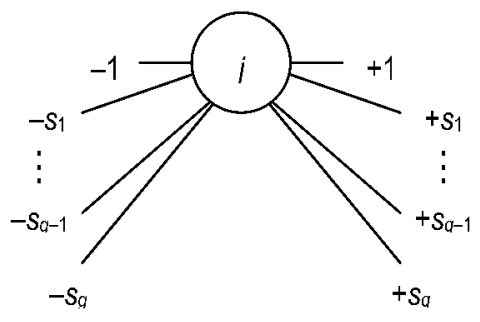

(a) Chordal ring

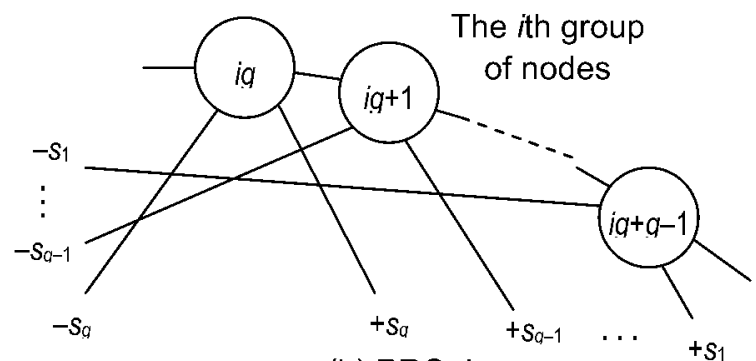

(b) PRC ring

Fig. 3. Node structure in chordal rings and PRC rings.

The construction leading to PRC rings is a special case of a node degree reduction strategy in which a node of degree $d$ is replaced by a network of lower-degree nodes so that the nodes are interconnected to each other and collectively provide $d$ external links to serve as the original links of the replaced node. Figure 4 shows how double-ring networks and PRC networks represent two different strategies for applying this principle: a double-ring network is obtained when each ring node is replaced by a 2-node network in the radial direction, whereas a PRC ring network is obtained when substitution is in the tangential direction.

Chordal rings of node degree 3 have also been extensively studied, but they do not satisfy the definition given above for chordal rings. In degree-3 chordal rings, the skip distance alternates between $+s_{1}$ (for even nodes) and $-s_{1}$ (for odd nodes). Because each node only has a single skip link in the forward or backward direction, the node degree of 3 is ensured. Because of the way such networks are defined, they necessarily have an even number $p=2 q$ of nodes and an odd skip distance $s$ in forward and backward directions. These networks must in fact be categorized as periodically regular, with a group size of $g$ $=2$ and skips $+s$ and $-s$. We denote such a PRC ring of degree 3 as $P R C_{3}(p ; s)$. 
Periodically regular ring structures can also be used to characterize other well-known interconnection architectures [23].

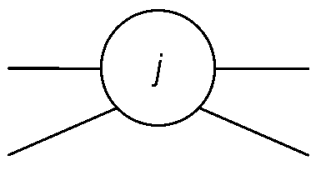

(a) Original

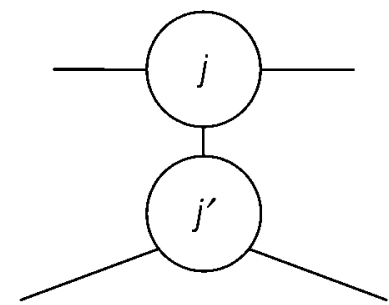

(b) Double-ring

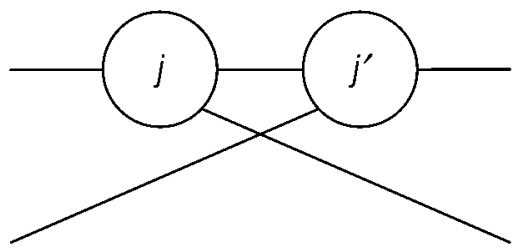

(c) PRC ring

Fig. 4. Two ways of replacing a degree-4 node with two degree-3 nodes.

\section{Double-Ring Networks as PRC Rings}

We begin by examining the simple double ring network $D R(12 ; 1)$ of Fig. 5a. From graph theory results, we know that this network is Hamiltonian. For example, a spanning cycle may consist of any two adjacent spoke edges, plus all but one of the outer and inner edge sets. Tracing a different type of Hamiltonian cycle that moves between the outer and inner rings, taking one step in each before switching to the other, leads to an alternate drawing of the network, as shown in Fig. 5b. The PRC network isomorphic to the double-ring network of Fig. 5a has a group size of $g=2$ and skip distances of +3 for even nodes and -3 for odd nodes. Clearly, it is possible to use the same construction for any $\operatorname{DR}(n ; 1)$ with $n$ even. It remains to establish whether an isomorphic PRC ring can be found when $n$ is odd. The following theorem shows that the answer is negative.

Note that in the double-ring network of Fig. 5a, spoke edges are different from other edges (the network is not edge-transitive). To see this property, which we will use in the proof of Theorem 1, consider that each spoke link is part of two different cycles of length 4 , whereas each outer or inner link belongs to only one such cycle. Figure 6 shows the interchangeability of outer and inner links for $D R(12 ; 5)$.

Theorem 1: A simple double-ring network $D R(n ; 1)$ is a PRC ring if and only if $n$ is even.

Proof: The "if" part is straightforward, given the construction discussed above (leading to the redrawing of Fig. 5a as Fig. 5b). To prove the "only if" part, let $n$ be odd. Clearly, we cannot trace a Hamiltonian cycle by alternating between the outer and inner rings, switching on every other step, as done in the aforementioned construction. Any Hamiltonian cycle must thus take a hop somewhere from the outer/inner to the 
inner/outer loop, remaining there for 2 or more hops. Without loss of generality, let the path go from node 0 to $0^{\prime}$ and from there to $1^{\prime}$ and then $2^{\prime}$. It is easy to see that the postulated Hamiltonian cycle cannot be completed, except as follows:

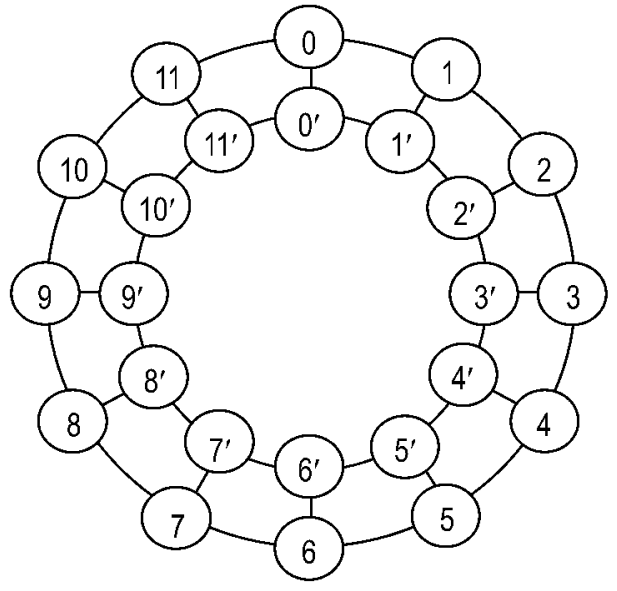

(a) Simple double-ring network $G P(12 ; 1)$

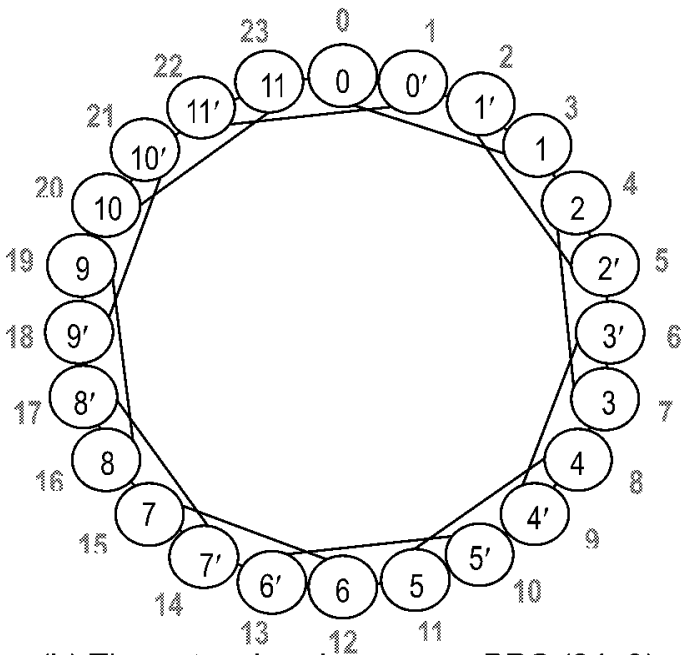

(b) The network redrawn as a $P R C_{3}(24 ; 3)$

Fig. 5. The double-ring network and its chordal ring view.

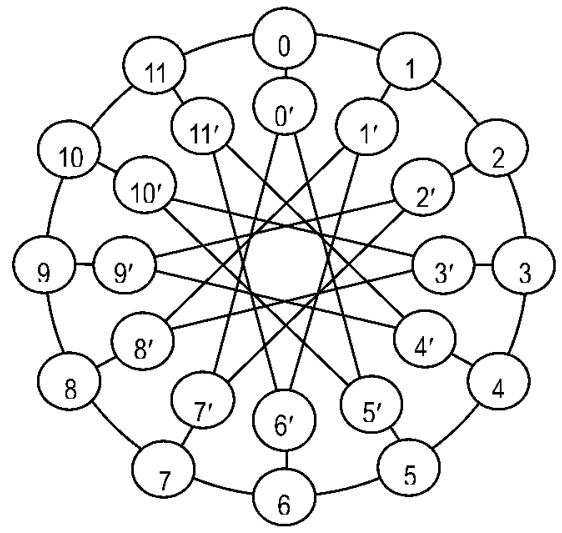

(a) The original double-ring network

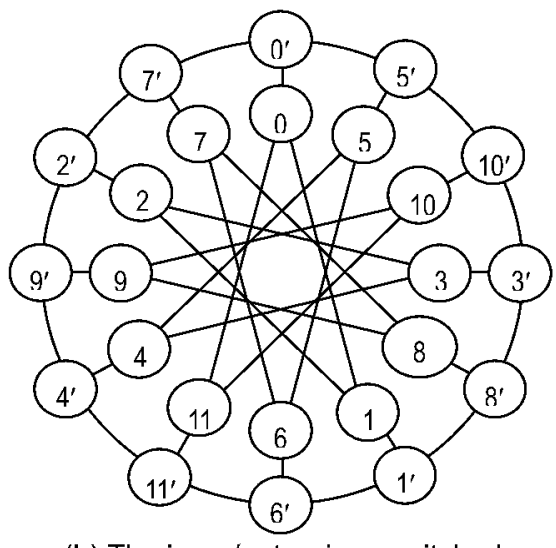

(b) The inner/outer rings switched

Fig. 6. Switching the outer and inner rings in the double-ring network $D R(12 ; 5)$.

$\begin{array}{lllllllllllll}0 & 0^{\prime} & 1^{\prime} & 2^{\prime} & \ldots & (n-2)^{\prime} & (n-1)^{\prime} & (n-1) & (n-2) & \ldots & 2 & 1 & 0\end{array}$

Thus, only two spoke links are part of the Hamiltonian path. Because these links are different from (not interchangeable with) the outer and inner links, as discussed previously, it is impossible for them to be part of a PRC ring with a 
period that is less than $n / 2$. Note that in a PRC ring, any two skip links of the same type $s_{i}$ that are separated by $g$ nodes (i.e., they emanate from nodes $j$ and $j+g$, where $g$ is the period) are completely interchangeable. !

For generalized double-ring networks, the construction is somewhat more complicated. Consider for example the $D R(12 ; 3)$ network in Fig. 7a. To trace a Hamiltonian path in this network in a way that it produces an isomorphic PRC ring, spoke links must be utilized at equal intervals. Note that this is a necessary, but not sufficient, condition; it is necessary because periodicity (with a period of $g$ ) dictates that links emanating from nodes $j$ and $j+h g$ be interchangeable. One possibility is shown in Fig. $7 \mathrm{~b}$, which is a PRC ring with $g=4$ and skip distances $-5,-11,+11,+5$.

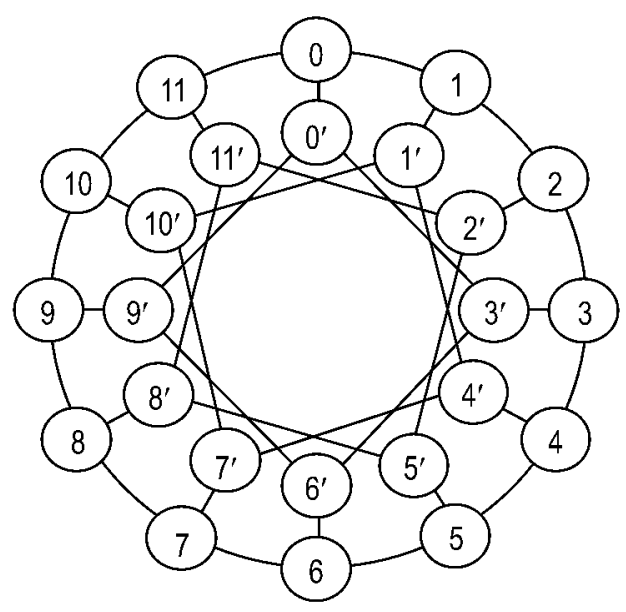

(a) Generalized Petersen graph, $G P(12 ; 3)$

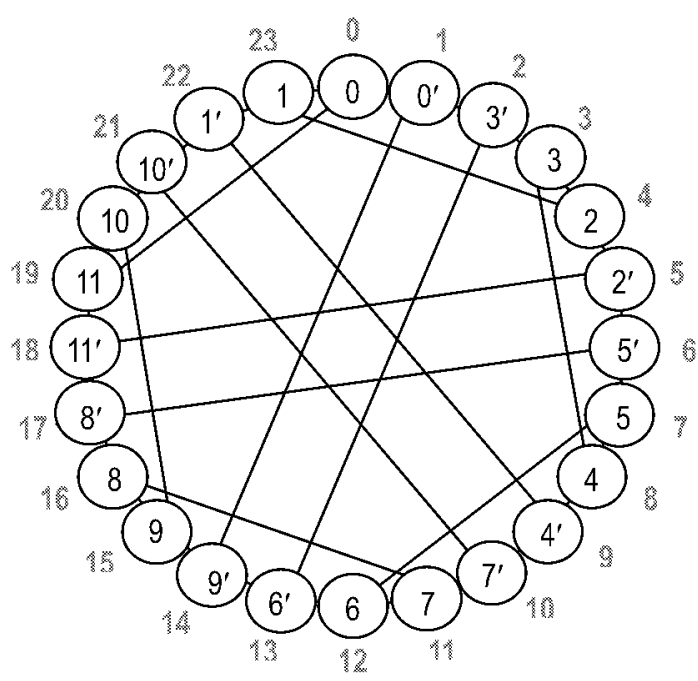

(b) $\mathrm{GP}(12 ; 3)$ redrawn as a PRC ring

Fig. 7. A generalized Petersen graph and its periodically regular chordal ring view.

Theorem 2: $G P(n ; s)$ is a PRC ring if $n$ is even, $s>1$ is odd, and $n=2 \bmod (s-1)$.

Proof: Note that the condition " $n$ even" is redundant, and thus could have been left out, because it is implied by " $s$ odd" and $n=2 \bmod (s-1)$. Begin a Hamiltonian path at 0 . Proceed as follows, taking spoke links on alternate steps:

$$
\begin{array}{lllllllllll}
0 & \underline{0}^{\prime} & s^{\prime} & s & (s-1) & \underline{(s-1)^{\prime}} & (2 s-1)^{\prime} & (2 s-1) & (2 s-2) & \underline{(2 s-2)^{\prime}} & \ldots
\end{array}
$$

This pattern in the partial sequence shown above is periodic, with a period of 4 , as evidenced by the underlined node numbers that are multiples of $s-1$ and on the inner ring. If continuation of the underlined sequence of node numbers leads to the node $(s-2)^{\prime}$, the pattern can be repeated by proceeding to $(s-2),(s-3)$, and $(s-3)^{\prime}$. From there, each 
node number is shifted by -2 (modulo $n$ ) relative to those in the previous repetition, eventually closing the path via the final nodes $1^{\prime}$ and 1 . Thus, because of the wraparound when going beyond the starting nodes 0 and $0^{\prime}$, some multiple of $s-1$ must equal $n+s-$ 3 , that is, $l(s-1)=n+s-3$. To make the right-hand side of the latter equation a multiple of $s-1$, we must have $n=2 \bmod (s-1)$. Note that in the special case of $s=3$, the latter condition becomes $n=2 \bmod 2$, which is the same as " $n$ even." !

Theorem 2 presents sufficient conditions for $D R(n ; s)$ to be a PRC ring. If the postulated conditions are satisfied, then the isomorphic PRC ring has a period of 4 . At this point, we do not know whether these conditions are necessary as well, although we strongly suspect that they are. Here is an informal argument suggesting the truth of our conjecture. Suppose we do not take a spoke edge on every other step along the Hamiltonian cycle. For example, let a Hamiltonian path begin in the following manner:

$\begin{array}{lllllllll}0 & 0^{\prime} & s^{\prime} & 2 s^{\prime} & \ldots & h s^{\prime} & h s & \ldots\end{array}$

That is, rather than alternating between the outer and inner rings, the path stays on the inner (or outer) ring for $h>1$ steps. Because with the choices above, nodes $s, 2 s, \ldots$, (h - 1)s are left with their spoke links unusable in our path construction, the postulated Hamiltonian cycle must include the path segments $s-1, s, s+1 ; 2 s-1,2 s, 2 s+1 ; \ldots$; $(h-1) s-1,(h-1) s,(h-1) s+1$. Experimentation with a few networks of varying sizes and structures quickly reveals the difficulty of completing the Hamiltonian cycle with these constraints. We stress that the argument above is not a proof; it is simply offered to reveal the intuition behind our conjecture that the sufficient conditions of Theorem 3 are also necessary.

The final condition on $n$ in the statement of Theorem 2 , that is, $n=2 \bmod (s-1)$, is not as restrictive as it appears at first glance. For example, it is always satisfied for $s=3$, it is satisfied for $s=5$ provided that $n$ is not a multiple of 4 , and, more generally, it holds for one out of every $(s-1) / 2$ consecutive even value of $n$. Therefore, a PRC ring network of size close to that of an arbitrary double-ring or generalized Petersen network can always be constructed. This notion is formalized in the following theorem.

Theorem 3: Given an arbitrary generalized Petersen network $G P(n ; s)$ with $n$ even, $s$ odd $(s \geq 5)$, and $n \neq 2 \bmod (s-1)$, we can construct a PRC ring with no more than $2(n+s-3)$ nodes whose diameter is at most one unit greater than that of the original network.

Proof: Let $n=m \bmod (s-1)$, where $m$ is even and $m \neq 2$. The PRC ring postulated is derived from the Hamiltonian-path construction of Theorem 2, after adding $2[(s+1-m)$ $\bmod (s-1)]$ nodes to $G P(n ; s)$ to transform it into $G P(n+(s+1-m) \bmod (s-1) ; s)$. Because $(s+1)=2 \bmod (s-1)$, it is readily seen that the number of nodes in the 
expanded network is $2 \bmod (s-1)$, thus making the Hamiltonian path construction method of Theorem 2 applicable. The maximum enlargement of $n$ during the transformation above is $s-3$ (occurs for $m=4$ ), leading to the maximum of $2(n+s-3)$ additional nodes in the network, as claimed. The fact that the diameter of the expanded network is no more than one unit greater than that of the original $G P(n ; s)$ network is readily provable by the observation that there can be no more than an extra skip link on the shortest path from any source node to a destination node, compared with a corresponding diametral path in the original network. !

\section{Practical Implications}

Based on Theorem 3, even when a given double-ring network is not isomorphic to a PRC ring, we can use a PRC ring of comparable size and diameter in lieu of the former. The replacement network also has identical fault tolerance and robustness attributes, given that it is a member of the same class of networks as the one it replaces. The relative size increase in this replacement is no greater than $(s-3) / n$. In general, the optimal value of $s$, that is, the skip value that minimizes the network diameter and its average distance, is $s^{\text {opt }}$ $=\Theta\left(n^{1 / 2}\right)$. Both smaller and larger skip values increase the diameter and its average distance compared with the optimal choice. Consequently, we can assume without any harm to performance attributes of the networks that $s=\mathrm{O}\left(n^{1 / 2}\right)$. The worst-case relative size increase from double-ring network to the corresponding PRC ring is thus $\mathrm{O}\left(n^{-1 / 2}\right)$. This is no worse than the effect of adding a single row or column to a $2 \mathrm{D}$ (nearly) square mesh or torus network. The effect on diameter is also comparable: expanding an odd side length of a torus by one, or adding a row/column to a mesh, causes a unit increase in diameter, while there is no change in diameter for incrementing an even side length in a torus.

If PRC rings are to be used in parallel architectures, either as direct choices or as preferable substitutes for double-ring networks, their structural properties must be contrasted with other fixed-degree networks of comparable implementation costs. For degree-3 PRC rings, the best-known competitors are shuffle-exchange permutation [25], cube-connected cycles [33], and pruned torus networks [24], [36]. In fact, PRC rings that replace double-ring networks constitute a class of pruned torus networks, though not the type previously studied. As depicted in Fig. 8a, any p-node PRC ring of degree 3 is isomorphic to an $s \times(p / s)$ pruned twisted torus, where $s$ is the skip distance. Figure $8 \mathrm{~b}$ shows that some PRC rings are isomorphic to ordinary pruned tori. Thus, documented advantages of pruned tori carry over to PRC rings, as do comparative evaluations between pruned tori and other fixed-degree networks. 
The next issue to be considered is that of incremental scalability, that is, the extent to which a network can be expanded to include a larger number of nodes. In this respect, hypercube networks are not very scalable for two different reasons: the fairly large step size in going from one size to the next possible size with twice as many nodes, and the increase in node degree to allow this expansion. Incomplete hypercubes [22] remedy the first problem, but not the second one. Similarly, the incremental scalability of many fixed-degree derivatives of hypercube networks suffers from the requirement that the network size be a power of 2 . Ring and mesh networks, on the other hand, are more readily scalable. Even though the incremental scalability of PRC rings follows from the pruned twisted torus equivalence depicted in Fig. 8a, we can also observe it directly. Figure 9 shows a network expansion strategy that can be readily generalized to an arbitrary value of the skip distance $s$. In this generalized form, expansion by $s+1$ nodes requires the redirection of $s-1$ of the existing links. When $s$ is chosen optimally to be $\mathrm{O}\left(n^{1 / 2}\right)$, the incremental scalability of PRC rings is comparable to that of $2 \mathrm{D}$ tori. Given that torus networks are deemed highly scalable, the incremental scalability of PRC rings must also be rated quite good.
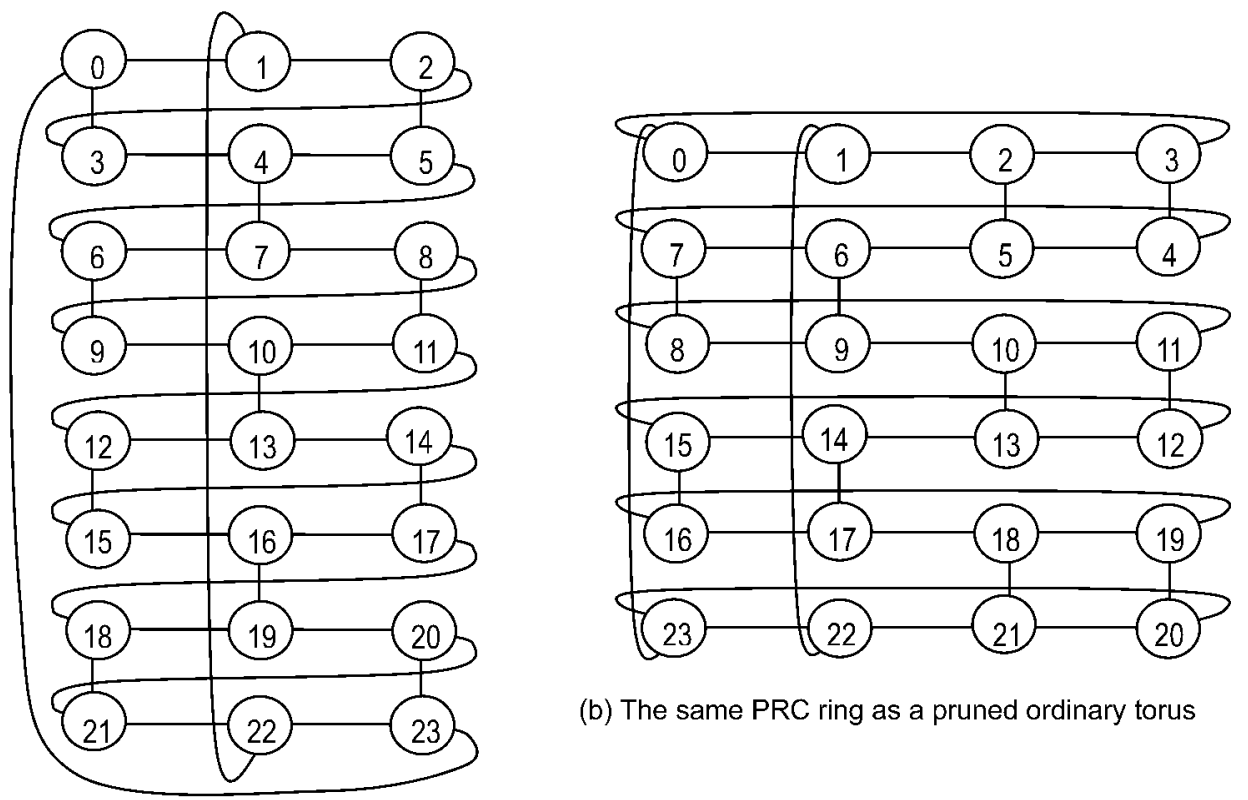

(b) The same PRC ring as a pruned ordinary torus

(a) A PRC ring as pruned twisted torus

Fig. 8. A PRC ring network and its representations as a pruned (twisted) torus.

We will show, in the next two sections, that problems of message routing (including wormhole switching, deadlock avoidance, and adaptation or fault tolerance) for degree-3 PRC rings are also comparable to, and at times simpler 
than, those of mesh and torus networks. This is not surprising, given the representations depicted in Fig. 8. Thus, from the preceding discussions and those to follow in Sections 6 and 7, we see that such degree-3 PRC rings can advantageously replace not only double-ring networks (owing to better structural properties), but also mesh/torus networks (because of lower node degree). Furthermore, such PRC rings are comparable to pruned tori, which have been previously studied and shown to have advantages over other regular networks [29].

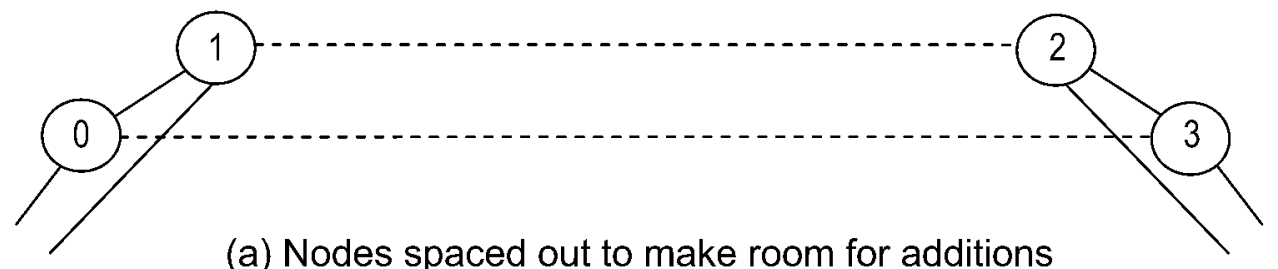

(a) Nodes spaced out to make room for additions

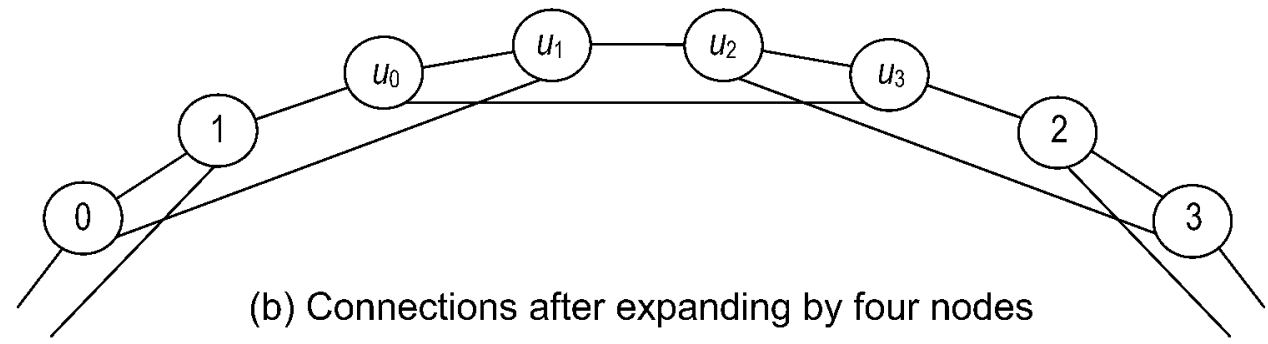

Fig. 9. Expanding a degree-3 PRC network with skip distance $s_{1}=3$.

Any class of networks that can replace, or show benefits over, multiple distinct classes of networks would be a useful addition to the repertoire of parallel computer architect. For example, it allows single streams of effort for algorithm development, provision of fault tolerance, analysis of energy efficiency, and packaging considerations to replace multiple current streams.

\section{Routing Algorithms}

We now turn to routing algorithms for degree-3 PRC ring networks, including issues of deadlock-freedom. Fault tolerance will be covered in Section 7. A natural first question in devising routing algorithms for a class of networks is whether shortest-path (optimal) routing is computationally feasible. For degree-3 PRC rings, the answer appears to be negative for now, although research is still continuing in this area. There are two reasons for this interim conclusion. First, although computationally efficient, $\mathrm{O}\left(\log ^{2} n\right)$-time, 
shortest-path routing algorithms can be adapted from those for double-ring networks [15], the overhead of carrying along information about a path precomputed at the source node or, alternatively, computing the path incrementally at each intermediate node appears to be too great for modern wormhole routing schemes that rely on extremely fast switching. Intuitively, the reason why the simple row-column scheme of tori does not work here is that some "column" links are missing and some links (row wraparounds in Fig. 8a) lead to movement in both horizontal and vertical directions. Second, even if we deemed the overhead of shortest-path computation tolerable, we would still have difficulties with deadlock avoidance (due to movements in both directions in a complicated, interlaced pattern that differs depending on the source-destination distance) as well as with provision for fault-tolerant routing.

Fortunately, the present impracticality of shortest-path routing for degree-3 PRC rings does not doom these networks. In the rest of this section, we show that elegant, near-optimal routing algorithms, with small, guaranteed bounds on the additional path length, exist for these networks. Furthermore, the algorithms allow simple deadlock avoidance and adaptation for fault tolerance.

Before introducing our routing algorithms, we present a representational tool that is useful in the design, understanding, and analysis of such algorithms. Given the PRC ring $P R C_{3}(n ; s)$, we can construct the infinite triangular grid shown in Fig. 10a, where each triangle is assigned a label in $[0, n-1]$ and the label increases by $1(\bmod n)$ when we move to the northeast or southeast neighbor and by $s(\bmod n)$ when we move to the north neighbor. Note that the 20-tile blocks defined by the heavy borders in Fig. 10a tessellate the plane, while holding each of the labels 0 to 19 exactly once. This correspondence between degree-3 PRC rings and infinite hex grids has been used before (see, e.g., [4]).

Figure $10 \mathrm{~b}$ depicts shortest paths from node 0 to various nodes in $P R C_{3}(20 ; 5)$. Of course, shortest paths are in most cases nonunique, and Fig. $10 \mathrm{~b}$ represents a particular arbitrary choice in such cases.

Infinite grids of various kinds (hex, honeycomb, square) have been applied to a number of problems in graph theory and interconnection networks. There is an interesting relationship between hex and square grid types in the case of degree-3 PRC rings. Figure 11 shows that by pairing each even-indexed node and the following odd-indexed node into a supernode, the structure of $P R C_{3}(20 ; 5)$ can be represented by a square grid which is identical to that of a degree- 4 chordal ring. Some of the adjacencies between supernodes (by virtue of links between their component nodes) have been marked by arrows in Fig. 11. We see that the horizontal and vertical grid directions in Fig. 11 correspond to the \pm 1 and $\pm s$ links in the degree- 4 chordal ring formed by the supernodes. 


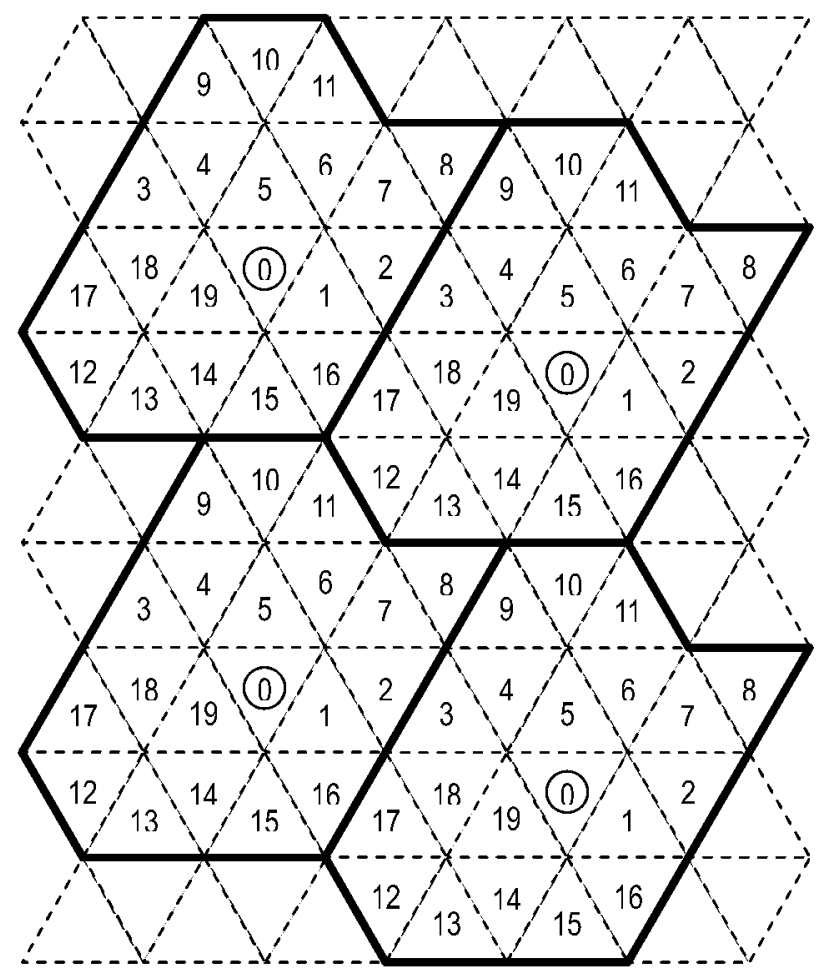

(a) Plane tessellation corresponding to CR3(20,5)

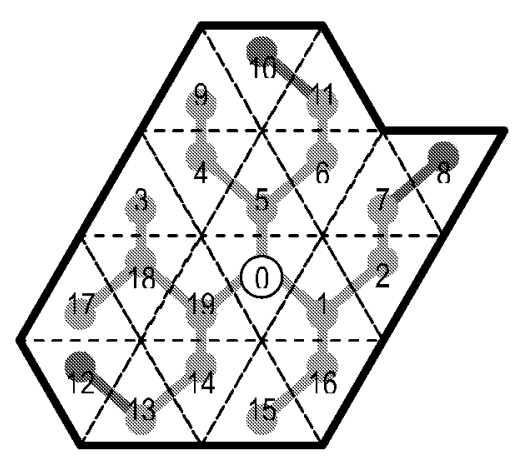

(b) Shortest-path routing

Fig. 10. The corresponding plane tessellation for a degree-3 PRC ring, and associated shortest paths.

\begin{tabular}{|c|c|c|c|c|c|}
\hline 6 & 8 & 10 & 12 & 14 & 16 \\
7 & 9 & 11 & 13 & 15 & 17 \\
\hline 2 & 4 & 6 & 8 & 10 & 12 \\
3 & 5 & 7 & 9 & 11 & 13 \\
\hline 18 & 0 & 2 & 4 & 6 & 8 \\
19 & 1 & 3 & 5 & 7 & 9 \\
\hline 14 & 16 & 18 & 0 & 2 & 4 \\
\hline 15 & 17 & 19 & 1 & 3 & 5 \\
\hline 10 & 12 & 14 & 16 & 18 & 0 \\
11 & 13 & 15 & 17 & 19 & 1 \\
\hline 6 & 8 & 10 & 12 & 14 & 16 \\
\hline 7 & 9 & 11 & 13 & 15 & 17 \\
\hline 2 & 4 & 6 & 8 & 10 & 12 \\
3 & 5 & 7 & 9 & 11 & 13 \\
\hline \multicolumn{7}{|c|}{} \\
\hline
\end{tabular}

Fig. 11. Square grid representing $P R C_{3}(20 ; 5)$ as a network of 10 supernodes. 
In what follows, we describe two distributed routing algorithms that provide the next node en route to node 0 . Algorithm 1 restricts the message movement to one direction around the ring (clockwise or counterclockwise) and, in so doing, leads to somewhat longer paths in some cases. We will see later that deadlock avoidance is simpler for Algorithm 1, thus making it the preferred candidate when $s$ is not large. Algorithm 2 routes messages in two phases: Phase 1 is unidirectional and uses both ring links and skip links, while Phase 2 uses ring links in the direction opposite to that of Phase 1. This allows the establishment of a tighter bound on the extra path length relative to optimal routing.

Algorithm 1: Unidirectional distributed routing on degree-3 PRC rings from node $i$ to node 0

if $i \geq n / 2$

then if $i$ is even and $i \leq n-s$

then send the message along the skip link to node $i+s$

else send it along the ring link to node $i+1$

endif

else if $i$ is odd and $i \geq s \quad$ [Note: $i<n / 2$ ]

then send the message along the skip link to node $i-s$

else send it along the ring link to node $i-1$

endif

endif !

Algorithm 2: Two-phase distributed routing on degree-3 PRC rings from node $i$ to node 0

if $i \geq n / 2$

then if $i$ is even and $i<n-\lceil s / 2\rceil$

then send the message along the skip link to node $i+s$

else send it along the ring link to node $i+1$

endif

else if $i$ is odd and $i>\lceil s / 2\rceil \quad$ [Note: $i<n / 2]$

then send the message along the skip link to node $i-s$

else send it along the ring link to node $i-1$

endif

endif !

Before deriving key properties of Algorithms 1 and 2, we need the following result.

Lemma 1: In a shortest path from node $u$ to node $v$, skip links are always taken in the same direction (forward or backward, but not both). 
Proof: Let there be some forward skip links on a shortest path from $u$ to $v$ and at least one backward skip link. If we list all the skip links taken in order, there must be at least one transition from forward to backward skip (or vice versa). We consider the first case only, because the proof for the second case is similar. Let $(2 a, 2 a+s)$ be the last forward skip link taken before the backward link $(2 b+1,2 b+1-s)$. The intermediate steps between the aforementioned skip steps correspond to ring links in the same direction (forward or backward), as shown in Fig. 12. It is readily seen that in both cases, the distance between nodes $2 a$ and $2 b+1-s$ is shorter than the length of the path segment shown, thus contradicting the assumption that the segments belong to a shortest path. !

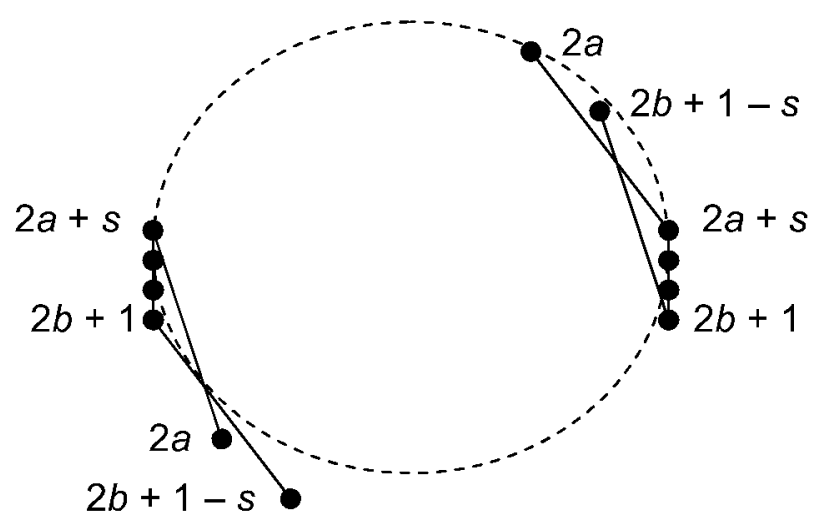

Fig. 12. The two subcases in the proof of Lemma 1.

Theorem 4: The unidirectional routing scheme defined by Algorithm 1 is near-optimal, that is, the path chosen by the algorithm is no more than $s-3$ hops longer than a shortest

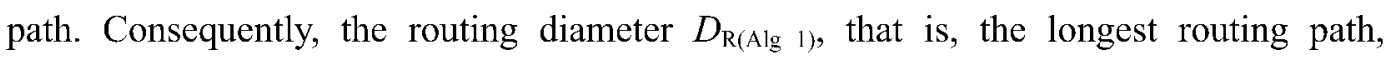
associated with Algorithm 1 is no greater than $D+s-3$, where $D$ is the network diameter.

Proof: We begin by observing that a shortest path from node $i$ to node 0 may have the following general form, where the pattern applies for $i$ even and $i \geq n / 2$ (the other three cases follow by symmetry).

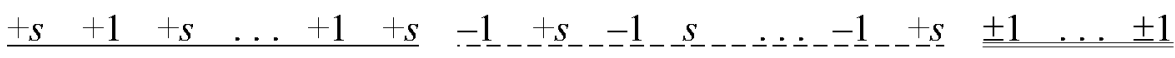

In other words, the path contains three segments: (1) The solid-underlined initial segment, consisting of $h(h \geq 0)$ skip links interlaced with $h-1$ forward ring links when $h \geq 2$; (2) The broken-underlined middle segment consisting of $k$ repetitions of the pattern $-1+s$; (3) 
The double-underlined final segment consisting of 0 or more ring links, all in the same direction. It is easy to see that if we have a shortest path in which the $+s+1$ and $-1+s$ patterns do not occur in the order shown above, we can easily derive another shortest path where the order follows the given pattern. Note that the use of skip links in only one direction is justified by Lemma 1. Now, the path chosen by Algorithm 1 is identical to the shortest path above in the initial segment. In the second segment, the path of Algorithm 1 diverges from the above by following the $+1+s$ pattern in lieu of $-1+s$. Given that each repetition of the new pattern takes us further towards the destination node, the maximum number of $+s$ links in the new pattern will be $k-1$ instead of $k$ assumed for the original pattern. The distance spanned in segment 2 as a result of following the original pattern is $k(s-1)$ and the number of hops taken is $2 k$. For the pattern of Algorithm 1, the distance spanned is $(k-1)(s+1)$ and the number of hops taken is $2 k-2$. Thus, the node reached by Algorithm 1 at this juncture is $k(s-1)-(k-1)(s+1)=s-2 k+1$ ring hops short, but the algorithm has taken 2 fewer hops up to this point. The penalty (extra routing hops) for the algorithm is thus no greater than $(s-2 k+1)-2=s-2 k-1$ hops. Of course, there is no penalty, or extra hops, when $k=0$, so an upper bound on the number of extra hops taken by Algorithm 1 is $s-3$ (occurring for $k=1$ ). Note that we ignored the third segment of the shortest path shown above, because it is immaterial if it consists of +1 steps and will reduce the penalty if it consists of -1 steps. !

Theorem 5: The two-phase routing scheme defined by Algorithm 2 is near-optimal, that is, its chosen path is no more than $\lfloor s / 2\rfloor$ hops longer than a shortest path. Consequently, the routing diameter $D_{\mathrm{R}(\mathrm{Alg} 2)}$ associated with Algorithm 2 is no greater than $D+\lfloor s / 2\rfloor$, where $D$ is the network diameter.

Proof: The proof is very similar to that of Theorem 4, so we skip most of the details. Let the following be a shortest path from node $i$ to node 0 , with its three segments defined in the proof of Theorem 4 .

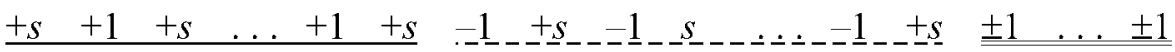

Again, we can ignore the first and third segments, as previously argued. Because Algorithm 2 differs from Algorithm 1 only in the way that the final phase of routing is handled (i.e., it chooses the shorter of the same-direction or reverse ring hops to finish the routing), $s-2 k-1$ derived in the proof of Theorem 4 is still an upper bound on the number of extra hops. A second upper bound can be obtained by assuming that the $+1+s$ patterns of Algorithm 2, repeated $k$ times for the second segment, take us past the destination node, where reverse routing takes effect. This intermediate node is $k(s+1)$ hops away from the beginning node of the middle segment, thus needing up to $k(s+1)-$ 
$k(s-1)=2 k$ reverse routing steps. The overall penalty in terms of extra hops is thus $\min (s$ $-2 k-1,2 k$ ), which is no greater than $\lfloor s / 2\rfloor$. !

Finally, we turn to the question of deadlocks when Algorithms 1 and 2 are used with wormhole switching. The unidirectional message flow of Algorithm 1 makes it amenable to conventional deadlock avoidance schemes used for simple ring networks. The only cause of circular waiting among messages send via Algorithm 1 is the wrapping around of message paths when crossing node 0 . Providing two virtual channels per link is adequate in this case; for an explanation, see (for example) Section 7 in [28]. In the case of Algorithm 2, we have the following result.

Theorem 6: The two-phase routing scheme defined by Algorithm 2 is deadlock-free when used with wormhole switching, provided messages in their reverse movement phase are given higher priority.

Proof: The reverse movement phase occurs near the completion of a message's routing path and is fairly short. If messages in the latter phase are given priority, simple ring routing guarantees their completion in a short time. The wait time for all other messages is thus finite, making deadlock impossible.

\section{Fault Tolerance}

Chordal rings are known to be maximally fault-tolerant. In other words, in a degree-3 chordal ring, there exist three node-disjoint paths between any pair of nodes. In graph theory literature, this property is known as the maximal connectivity of (connected) circulant graphs [37]. Figure 13 depicts examples, where three disjoint paths between node pairs $(0,1)$ and $(0,10)$ have been drawn using the infinite hex grid tool introduced in Section 6. What remains to establish in this section is a method for deriving these alternate paths and placing a bound on their lengths in an effort to analyze the performance of a fault-tolerant routing algorithm that takes advantage of such paths. Our main result for the case $s \geq 5$ is stated in Theorem 7, with the special case of $s=3$ discussed afterwards.

Theorem 7: For $s \geq 5$, three node-disjoint (parallel) paths exist from any node $i$ to node 0 of $P R C_{3}(n ; s)$, such that the paths can be identified by a simple modification to Algorithm 1 and the longest of the three paths takes no more than $s-1$ extra hops compared to the shortest path between the two nodes. 


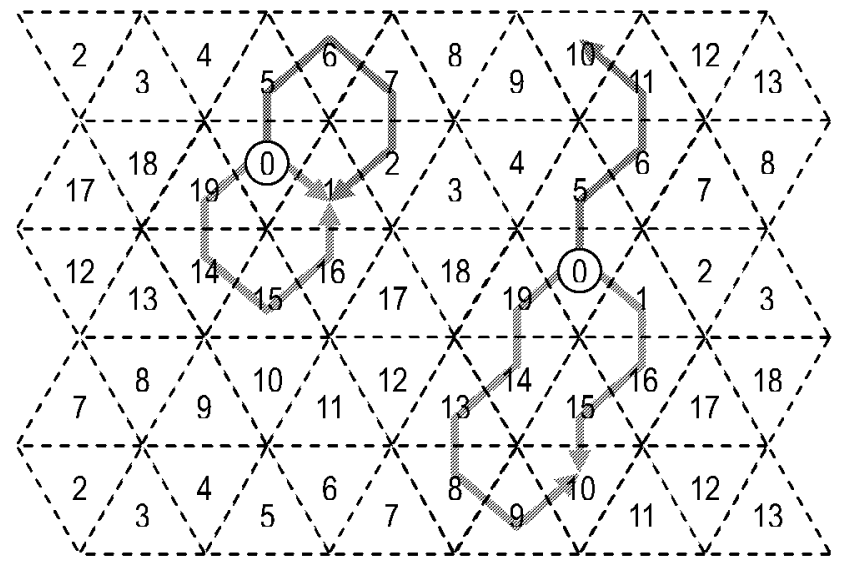

Fig. 13. Node-disjoint (parallel) paths between two node pairs in $P R C_{3}(20 ; 5)$.

Proof: Due to symmetry, we can restrict $i$ to the range $n / 2 \leq i \leq n-1$. First consider the case where Algorithm 1 uses a path with no skip links. The chosen path from $i$ to 0 thus consists of at most $s$ forward ring links: $i \rightarrow i+1 \rightarrow i+2 \rightarrow \ldots \rightarrow n-2 \rightarrow n-1 \rightarrow 0$. The three paths used in the two cases of $i$ even and $i$ odd are depicted in Figs. 14a and $14 \mathrm{~b}$, respectively, with alternate grid-based representations shown in Figs. 15a and 15b. In constructing these figures, we have assumed $n-i \geq 4$, but the proof in the remaining cases of $n-i=1,2$, or 3 is similar. In the case of Fig. 14a, the paths (labeled 1, 2, and 3 at their origins in node $i$ and termination in node 0$)$ are of lengths $n-i+2,(s+1)-(n-$ $i)$, and $(s+3)-(n-i)$, respectively. Owing to the assumption $n-i \geq 4$, the conclusion that the longest of these paths is no more than $s-1$ hops longer than the shortest path from $i$ to 0 is immediate. From the representation in Fig. 15a, we see that moving node $i$ in the same grid row will not affect the preceding result. In the case of Fig. $14 \mathrm{~b}$, the three path lengths are $n-i+2, n-i+2$, and $(s+1)-(n-i)$, respectively. Again we see that none of the lengths exceeds that of a shortest path by more than $s-1$ hops. We next turn to the case where at least one skip link is included in the path chosen by Algorithm 1. Let the path move from node $i$ to node $j$ and from that point on, use only forward ring links. Given that node $j$ is entered via a forward skip link on this path, it must be an odd-indexed node. If $i$ is even, the path from $i$ to $j$, defined in terms of the links taken, will be of the form ${ }_{s},+1,+s,+1, \ldots,+1,+s$. For odd $i$, the starting step would be +1 instead of $+s$, but the two step types alternate in the same way. Consider the first case, and call the string of steps taken $P$. The alternate paths $+1+1 P$ and $-1-1 P$ will lead to nodes $j+2$ and $j-2$ respectively, with the corresponding path segments being node-disjoint, given the assumption $s \geq 5$. From nodes $j-2, j$, and $j+2$, the three paths proceed to node 0 as depicted in Fig. 15c. Simple counting of the path lengths, as done for the precious 
cases, establishes the desired result. The case of $i$ odd can be handled similarly.

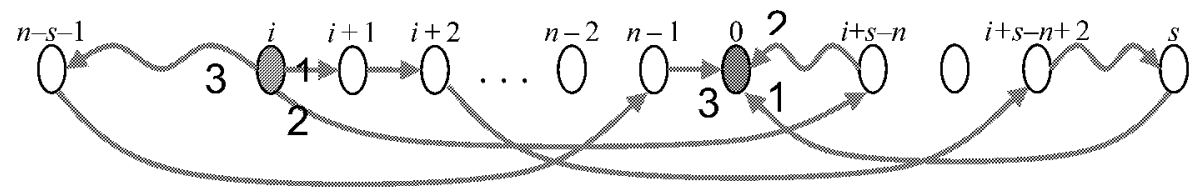

(a) The case of $i$ even

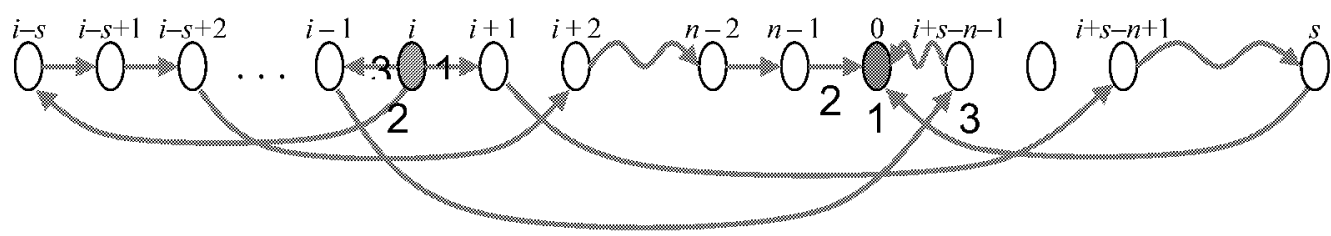

(b) The case of $i$ odd

Fig. 14. Three parallel paths in the case where Algorithm 1 would not use any skip links.

The following intuitive explanation helps us understand the constructions used in the proof of Theorem 7 and establishes why the case $s=3$ was excluded from the proof. As shown in Fig. 16, both the source node $i$ and the destination node 0 have all three of their links used within the postulated parallel paths. When node $i$ is not very close to node 0 , the three parallel paths proceed from node $i$ to the vicinity of node 0 , using interlaced segments that move in the same direction and consist of alternate $+s$ and +1 steps. Recall that $i$ is assumed to be $n / 2$ or greater; the steps would be $-s$ and -1 for counterclockwise paths associated with $i<n / 2$. For $s=3$, only two such interlaced paths can be constructed, with the third parallel path is forced to go in the opposite direction on the ring. Now, if $n$ is large and $i$ is approximately $3 n / 4$, this third alternate path will be much longer (almost 3 times as long, when $s<<n$ ) than the two near-optimal paths going in the same direction. Hence, no useful bound can be placed on the length of the longest of the three parallel paths in the case of $s=3$.

\section{Conclusion}

We have shown that many (generalized) double-ring networks and generalized Petersen graphs are isomorphic to periodically regular chordal rings. Additionally, we have demonstrated that when a $D R(n ; s)$ or $G P(n ; s)$ network is not isomorphic to a PRC ring, it can be replaced by a PRC ring that is at most only slightly larger, has a diameter that is either the same or one unit more, and enjoys identical fault tolerance and robustness parameters. We are now studying the practical implications of these results for communications networks as well as in the context of parallel and distributed computing. 


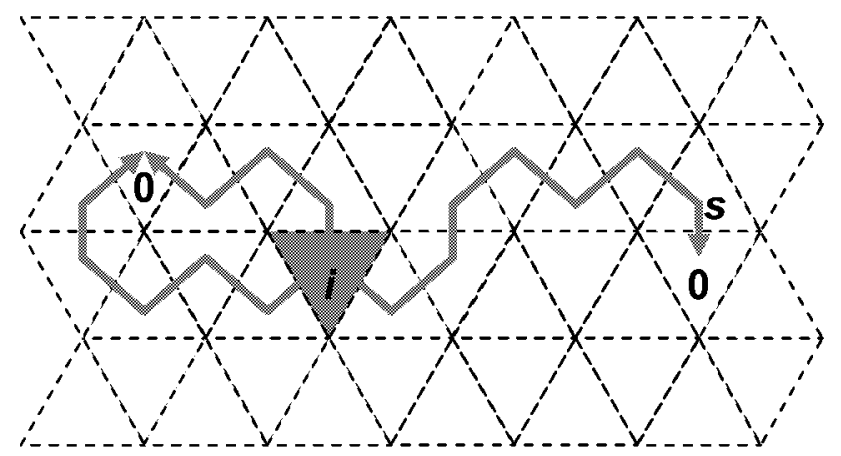

(a) Only ring hops on the original path (i even)

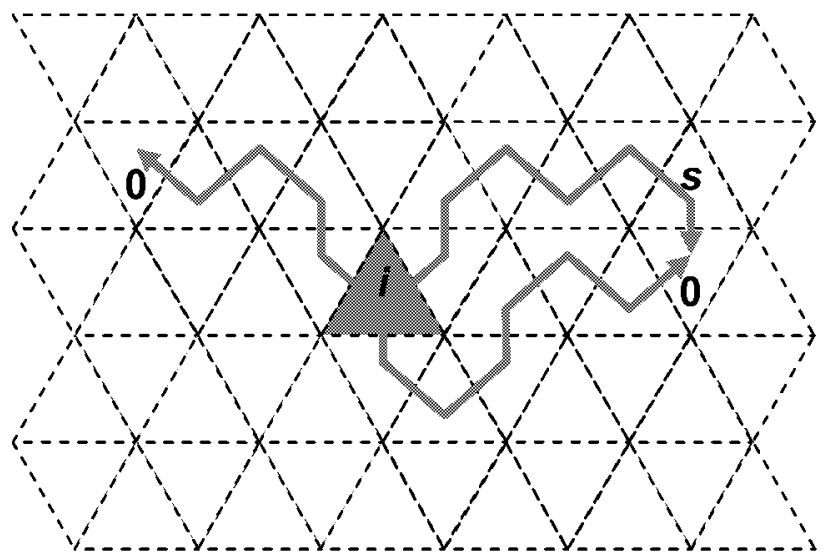

(b) Only ring hops on the original path (i odd)
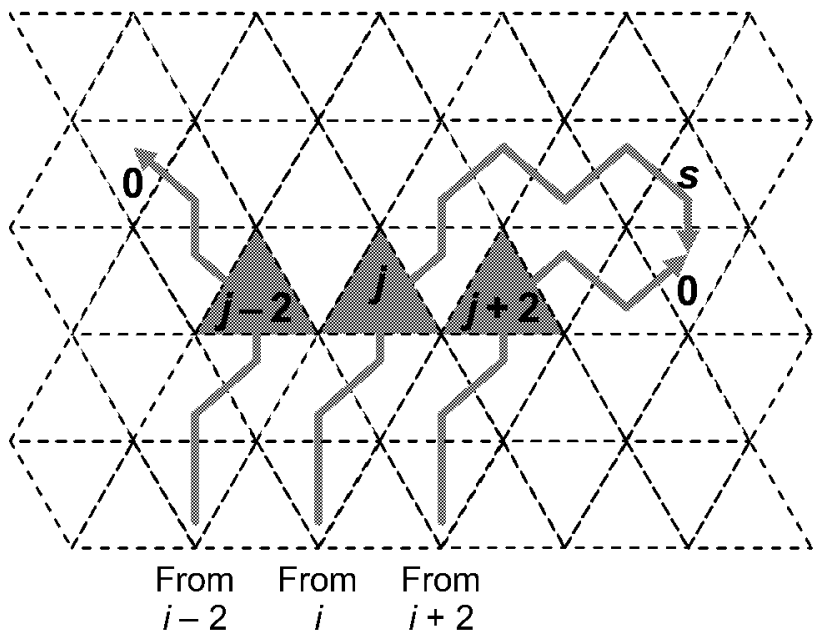

(c) The general case for $\mathrm{j}$ odd

Fig. 15. Node-disjoint (parallel) paths in the proof of Theorem 7. 


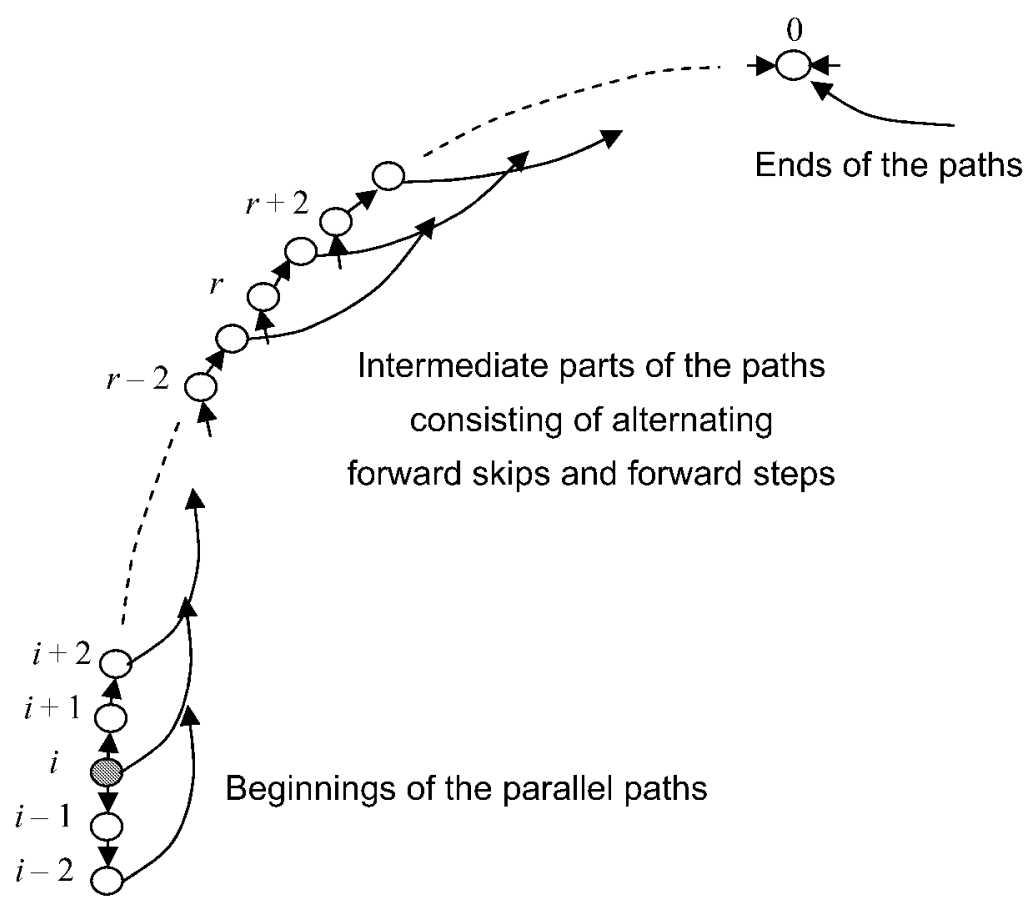

Fig. 16. Intuitive explanation of the parallel path of Theorem 7.

In the latter case, applications to intercluster communication and on-chip networks are of particular interest.

Alternate formulations and representations of particular interconnection structures have proven quite useful in the past. For double-ring networks, we note that routing and determination of alternate paths (including establishment of bounds on their lengths) become simpler with the PRC ring view. It is certainly an advantage for a number of routing functions to have a Hamiltonian cycle readily visible and not requiring any calculations to derive. We presented two near-shortest-path routing algorithm and showed how the simpler of the two can be adapted for fault-tolerant routing using alternate paths that are only $s-1$ or fewer hops longer than a shortest path. We are now working on simplifying the algorithms for shortest-path routing, derive sharper bounds on the lengths of node-disjoint parallel paths, and adapting other routing schemes (such as interval routing) to our networks.

Other areas for future research include the derivation of exact values (closed-form formulas) for the diameter, bisection width, and fault diameter of degree-3 PRC rings. Analyzing the performance of such networks under realistic message traffic patterns 
constitutes another fruitful area of research. There are preliminary indications that performance benefits do exist and could be significant in some cases. This is a direct result of the fact that our networks are quite similar to pruned torus networks that we have previously shown to be preferable to both ordinary torus networks and their unidirectional versions, known as Manhattan street networks, under most traffic conditions [29]. The node symmetry of PRC rings and their periodic regularity (the next best thing, in the absence of complete edge-symmetry) make both analytical and experimental evaluations feasible with moderate effort.

\section{References}

[1] B. R. Alspach, "The Classification of Hamiltonian Generalized Petersen Graphs," $J$. Combinatorial Theory, Series B, Vol. 34, pp. 293-312, 1983.

[2] B. W. Arden, and H. Lee, "Analysis of Chordal Ring Networks," IEEE Trans. Computers, Vol. 30, No. 4, pp. 291-295, April 1981.

[3] L. Barriere, J. Fabrega, E. Simo, and M. Zaragoza, "Fault-Tolerant Routings in Chordal Ring Networks," Networks, Vol. 36, No. 3, pp. 180-190, 2000.

[4] L. Barriere, "Symmetry Properties of Chordal Rings of Degree 3," Discrete Applied Mathematics, Vol. 129, pp. 211-232, 2003.

[5] R. Beivide, C. Martinez, C. Izu, J. Gutierrez, J.-A. Gregorio, and J. Miguel-Alonso, "Chordal Topologies for Interconnection Networks," Proc. 5th Int'l Symp. High-Performance Computing, October 2003, pp. 385-393.

[6] J.-C. Bermond, C. Delorme, and J.-J. Quisquater, "Tables of Large Graphs with Given Degree and Diameter," Information Processing Letters, Vol. 15, No. 1, pp. 10-13, 1982.

[7] J.-C. Bermond, C. Delorme, and J. Quisquater, "Table of Large $(\Delta, d)$-Graphs," Discrete Applied Mathematics, Vols. 37-38, pp. 575-577, 1992.

[8] J.-C. Bermond, F. Comellas, and D. F. Hsu, "Distributed Loop Computer Networks: A Survey," J. Parallel and Distributed Computing, Vol. 24, pp. 2-10, 1995.

[9] R. F. Browne, "The Embedding of Meshes and Trees into Degree Four Chordal Ring Networks," The Computer Journal, Vol. 38, No. 1, pp. 71-77, 1995.

[10] F. Buckley, and F. Harary, Distances in Graphs, Addison-Wesley, 1990.

[11] S. Bujnowski, B. Dubalski, and A. Zabludowski, "Analysis of 4th Degree Chordal 
Rings," Proc. International Conf. Communications in Computing, June 2004, pp. 318-324.

[12] N. Chalamaiah, and B. Ramamurty, "Finding Shortest Paths in Distributed Loop Networks," Information Processing Letters, Vol. 67, pp. 157-161, 1998.

[13] B. X. Chen, W. J. Xiao, and B. Parhami "Diameter Formulas for a Class of Undirected Double-Loop Networks,” J. Interconnection Networks, Vol. 6, No. 1, pp. 1-15, March 2005.

[14] D.-Z. Du, D. F. Hsu, Q. Li, and J. Xu, "A Combinatorial Problem Related to Distributed Loop Networks," Networks, Vol. 20, pp. 173-180, 1990.

[15] D. Gomez, J. Gutierrez, and A. Ibeas, "Optimal Routing in Double Loop Networks," Theoretical Computer Science, Vol. 381, pp. 68-85, 2007.

[16] W. D. Grover, Mesh-Based Survivable Networks: Options and Strategies for Optical, MPLS, SONET, and ATM Networking, Prentice Hall, 2004.

[17] D. A. Holton, and J. Sheehan, The Petersen Graph, Cambridge Univ. Press, 1993.

[18] R. M. Hord, The ILLIAC IV: The First Supercomputer, Springer-Verlag, Berlin, 1982.

[19] F. K. Hwang, “A Complementary Survey on Double-Loop Networks," Theoretical Computer Science, Vol. 263, pp. 211-229, 2001.

[20] F. K. Hwang, "A Survey on Multi-Loop Networks," Theoretical Computer Science, Vol. 299, Nos. 1-3, pp. 107-121, April 2003.

[21] T. Jorgensen, L. Pedersen, and J. M. Pedersen, "Reliability in Single, Double, and N2R Ring Network Structures," Proc. Int'l Conf. Communications in Computing, June 2005, pp. 189-195.

[22] H. P. Katseff, “Incomplete Hypercubes," IEEE Trans. Computers, Vol. 37, No. 5, pp. 604-608, May 1988.

[23] D.-M. Kwai, and B. Parhami, "A Generalization of Hypercubic Networks Based on Their Chordal Ring Structures," Parallel Processing Letters, Vol. 6, No. 4, pp. 469-477, 1996.

[24] D.-M. Kwai, and B. Parhami, "Pruned Three-Dimensional Toroidal Networks," Information Processing Letters, Vol. 68, No. 4, pp. 179-183, November 1998.

[25] S. Latifi, and P. K. Srimani, "A New Fixed Degree Regular Network for Parallel 
Processing," Proc. 8th IEEE Symp. Parallel and Distributed Processing, pp. 152-159, October 1996.

[26] M. Miller, and J. Siran, "Moore Graphs and Beyond: A Survey of the Degree/Diameter Problem," Electronic J. Combinatorics, \#DS14, 2005.

[27] K. Mukhopadhyaya, and B.P. Sinha, "Fault-Tolerant Routing in Distributed Loop Networks," IEEE Trans. Computers, Vol. 44, No. 12, pp. 1452-1456, 1995.

[28] B. Parhami, and D.-M. Kwai, "Periodically Regular Chordal Rings," IEEE Trans. Parallel and Distributed Systems, Vol. 10, No. 6, pp. 658-672, June 1999. (Printer errors corrected in Vol. 10, pp. 767-768, July 1999)

[29] B. Parhami, and D.-M. Kwai, "Comparing Four Classes of Torus-Based Parallel Architectures: Network Parameters and Communication Performance," Mathematical and Computer Modeling, Vol. 40, Nos. 7-8, pp. 701-720, 2004.

[30] B. Parhami, "Chordal Rings Based on Symmetric Odd-Radix Number Systems," Proc. Int'l Conf. Communications in Computing, June 2005, pp. 196-199.

[31] J. M. Pedersen, T. P. Knudsen, and O. B. Madsen, "Comparing and Selecting Generalized Double Ring Network Structures," Proc. Conf. Communication and Computer Networks, November 2004, pp. 375-380.

[32] J. M. Pedersen, T. M. Riaz, and O. B. Madsen, "Distances in Generalized Double Rings and Degree Three Chordal Rings," Proc. Conf. Parallel and Distributed Computing and Networks, February 2005, pp. 153-158.

[33] F. P. Preparata, and J. Vuillemin, "The Cube-Connected Cycles: A Versatile Network for Parallel Computation," Communications of the ACM, Vol. 24, No. 5, pp. 300-309, May 1981.

[34] C. S. Raghavendra, M. Gerla, and A. Avizienis, "Reliable Loop Topologies for Large Local Computer Networks," IEEE Trans. Computers, Vol. 34, No. 1, pp. 46-54, January 1985.

[35] C. S. Raghavendra, and J. A. Silvester, "A Survey of Multi-Connected Loop Topologies for Local Computer Networks," Computer Networks and ISDN Systems, Vol. 11, No. 1, pp. 29-42, January 1986.

[36] W. J. Xiao, and B. Parhami, "Structural Properties of Cayley Digraphs with Applications to Mesh and Pruned Torus Interconnection Networks," International J. Computer and System Sciences, to appear. 
[37] J. Xu, Topological Structure and Analysis of Interconnection Networks, Kluwer, 2001.

[38] Y. Yang, A. Funahashi, A. Jouraku, H. Nishi, H. Amano, and T. Sueyoshi, "Recursive Diagonal Torus: An Interconnection Network for Massively Parallel Computers," IEEE Trans. Parallel and Distributed Systems, Vol. 12, No. 7, pp. $701-715$, July 2001.

[39] J. A. L. Yenra, M. A. Fiol, P. Morillo, and I. Alegre, "The Diameter of Undirected Graphs Associated to Plane Tessellations," Ars Combinatoria, Vol. 20-B, pp. 151-171, 1985 .

[40] J. Zerovnik, and T. Pisanski, "Computing the Diameter in Multi-Loop Networks, $J$. Algorithms, Vol. 14, pp. 226-243, 1993. 